\title{
APPROXIMATING MULTIVARIATE TEMPERED STABLE PROCESSES
}

\author{
BORIS BAEUMER *** AND \\ MIHÁLY KOVÁCS, ${ }^{*}$ University of Otago
}

\begin{abstract}
We give a simple method to approximate multidimensional exponentially tempered stable processes and show that the approximating process converges in the Skorokhod topology to the tempered process. The approximation is based on the generation of a random angle and a random variable with a lower-dimensional Lévy measure. We then show that if an arbitrarily small normal random variable is added, the marginal densities converge uniformly at an almost linear rate, providing a critical tool to assess the performance of existing particle tracking codes.
\end{abstract}

Keywords: Tempered stable process; simulation; Lévy process; operator stable process; spectral measure

2010 Mathematics Subject Classification: Primary 60G51

Secondary 60G52

\section{Introduction}

Being able to approximate stable and tempered stable processes is important for investigation and simulation purposes. A stable or tempered stable process is a process whose increments are independent stable or tempered stable random vectors. An exponentially tempered stable random vector $X_{\rho}$ is obtained from a stable random vector $X$ by exponentially cooling its jump size (or Lévy measure); the general class of tempered stable vectors was introduced in [15]. Tempered stable laws are used in physics as a model for turbulent velocity fluctuations [11], [14], as well as in finance [2], [3] and hydrology [13], [16] as a model of transient anomalous diffusion [1]. As the random variables are infinitely divisible, they can be approximated using LePage's method, whereby their Lévy-Khintchine integral representation is split into a compound Poissonian part (with tempered Pareto jumps) and an approximately normal part [7]. For general processes with operator scaling, this is also nicely exhibited in [8]. In the case of a stable random vector this involves adding a random number of random vectors comprised of one-dimensional Pareto jumps multiplied by a random direction drawn according to the mixing measure $M$. In Zhang et al. [17] the authors compared this approach to just drawing a random direction multiplied by a one-dimensional skewed stable variable, which can also easily be generated [6]. As they are in the same domain of attraction of an operator stable [12], both approaches work well. However, in the case of tempered stable vectors, they are in the domain of attraction of the multivariate normal and, hence, another argument is needed. Furthermore, the rate of convergence was not explored; we address these issues in this paper.

In this paper we explore approximations to a stochastic process with stationary, independent increments for which we are given a coordinate transform $T: \Omega_{\theta} \times \Omega_{r} \rightarrow \mathbb{R}^{d}$ for some measure

Received 12 July 2011; revision received 13 October 2011.

* Postal address: Department of Mathematics and Statistics, University of Otago, PO Box 56, Dunedin, New Zealand.

** Email address: bbaeumer@maths.otago.ac.nz 
spaces $\Omega_{\theta}$ and $\Omega_{r}$ which decomposes the Lévy measure $\phi$ of the stochastic process into lowerdimensional Lévy measures; that is, assume that we have a probability measure $M$ on $\Omega_{\theta}$ and, for each $\theta \in \Omega_{\theta}$, there is a measure $\phi_{\theta}$ on $\Omega_{r}$ such that, with $\mathbf{1}_{A}$ being the indicator function of a set $A$,

$$
\begin{aligned}
\int \mathbf{1}_{A}(x) \phi(\mathrm{d} x) & =\int_{\Omega_{\theta} \times \Omega_{r}} \mathbf{1}_{A}(T(\theta, r)) \phi(\mathrm{d} T(\theta, r)) \\
& =\int_{\Omega_{\theta}} \int_{\Omega_{r}} \mathbf{1}_{A}(T(\theta, r)) \phi_{\theta}(\mathrm{d} r) M(\mathrm{~d} \theta)
\end{aligned}
$$

for any measurable set $A$ and the induced degenerate measure on $\mathbb{R}^{d}$ via

$$
\tilde{\phi}_{\theta}(A):=\int_{\Omega_{r}} \mathbf{1}_{A}(T(\theta, r)) \phi_{\theta}(\mathrm{d} r)
$$

is also Lévy. Note that for most processes appearing in applications there is a canonical decomposition.

We show that, for $\tau \rightarrow 0$, the processes

$$
S_{\tau}(t)=\sum_{j=1}^{\lfloor t / \tau\rfloor} X_{\Theta^{j}}^{j}(\tau)
$$

converge to the original stochastic process in the Skorokhod topology, where the increments $X_{\Theta^{j}}^{j}(\tau)$ are distributed according to the infinitely divisible distributions with Lévy measure $\tau \tilde{\phi}_{\Theta^{j}}$ and the $\Theta^{j}$ are random vectors distributed according to $M$. Here $\lfloor t / \tau\rfloor$ denotes the integer part of $t / \tau$. In the case that $\Omega_{r} \subset \mathbb{R}$ is a set of scale factors (radii), $\Omega_{\theta} \subset \mathbb{R}^{d}$ is a $(d-1)$-dimensional hypersurface (directions) and $T(\theta, r)=\theta r$, the increments take the simpler form $X_{\Theta^{j}}^{j}(\tau)=\Theta^{j} r_{\Theta^{j}}$, where $r_{\Theta^{j}}$ is a random variable with Lévy measure $\tau \phi_{\Theta^{j}}$ and $\Theta_{j}$ is a random direction picked according to $M$.

In particular, if $\Omega_{\theta}=\{\theta:\|\theta\|=1\}, \Omega_{r}=\mathbb{R}$, and if $\phi_{\theta}$ is the Lévy measure of a onedimensional tempered stable, this provides a method to approximate multidimensional tempered stable processes, extending the one-dimensional method to obtain tempered stable laws as random walk limits developed recently by Chakrabarty and Meerschaert [5].

We then investigate the rate of convergence in the exponentially tempered stable case with a single $\alpha$ [15] and show that the densities at a fixed time $t$ converge in the $L^{2}$-multiplier norm at a rate of $C\left(1+\log ^{2} n\right) / n$. We further show that, given a small random initial perturbation, the marginal densities of the processes converge uniformly in $x$ and $t$ at an almost linear rate. This is important as it provides a performance measure/benchmark for existing particle tracking codes [16], where this approximation method was used to estimate the density of the tempered stable model (without knowing how accurate the density approximation was), comparing it to a numerical simulation of particle transport in a random fracture network.

Furthermore, we also show that if the Lévy measure can be decomposed into finitely many tempered stable measures (e.g. of different $\alpha$ s) then, for each step, randomly choosing which tempered $\alpha$-stable to simulate also converges at an almost linear rate.

\section{The general result}

Let $X(t)$ be a stochastic process in $\mathbb{R}^{d}$ with stationary, independent increments. Then $X(t)$ has a unique Lévy-Khintchine representation,

$$
\mathrm{E}[\exp (-\mathrm{i}\langle k, X(t)\rangle)]=\exp (t \psi(k)),
$$


and $\log$-characteristic function,

$$
\psi(k)=\mathrm{i}\langle k, v\rangle-\frac{1}{2}\langle k, Q k\rangle+\int_{\mathbb{R}^{d}}\left(\mathrm{e}^{-\mathrm{i}\langle k, x\rangle}-1+\frac{\mathrm{i}\langle k, x\rangle}{1+\|x\|^{2}}\right) \phi(\mathrm{d} x)
$$

for some drift vector $v$, covariance matrix $Q$, and measure $\phi$ satisfying

$$
\int \frac{\|x\|^{2}}{1+\|x\|^{2}} \phi(\mathrm{d} x)<\infty .
$$

The notation $\langle x, y\rangle$ denotes the $\mathbb{R}^{d}$ inner product. Such a measure is called a Lévy measure.

Definition 1. Assume that, for a Lévy measure $\phi$, there exist measure spaces $\Omega_{\theta}$ and $\Omega_{r}$, and a measurable map $T: \Omega_{\theta} \times \Omega_{r} \rightarrow \mathbb{R}^{d}$ such that

$$
\int \mathbf{1}_{A}(x) \phi(\mathrm{d} x)=\int_{\Omega_{\theta}} \int_{\Omega_{r}} \mathbf{1}_{A}(T(\theta, r)) \phi_{\theta}(\mathrm{d} r) M(\mathrm{~d} \theta)
$$

for any measurable set $A$, and assume that the induced degenerate measure $\tilde{\phi}_{\theta}$ defined via $\tilde{\phi}_{\theta}(A)=\int_{\Omega_{r}} \mathbf{1}_{A}(T(\theta, r)) \phi_{\theta}(\mathrm{d} r)$ is Lévy. We then call $\left(\phi_{\theta}, M\right)$ a Lévy decomposition of $\phi$ and we call $\tilde{\phi}_{\theta}$ the projected Lévy measure in the direction of $\theta$.

Let $\Theta$ be an $\Omega_{\theta}$-valued random variable with $\operatorname{Pr}\{\Theta \in A\}=M(A)$. Let $X_{\theta}(\tau)$ be a random variable with characteristic function

$$
\mathrm{E}\left[\exp \left(-\mathrm{i}\left\langle k, X_{\theta}(\tau)\right\rangle\right)\right]=\exp \left(\tau \psi_{\theta}(k)\right)
$$

and

$$
\psi_{\theta}(k)=\mathrm{i}\langle k, v\rangle-\frac{1}{2}\langle k, Q k\rangle+\int_{\mathbb{R}^{d}}\left(\mathrm{e}^{-\mathrm{i}\langle k, x\rangle}-1+\frac{\mathrm{i}\langle k, x\rangle}{1+\|x\|^{2}}\right) \tilde{\phi}_{\theta}(\mathrm{d} x) .
$$

Clearly, $\psi(k)=\int_{\Omega_{\theta}} \psi_{\theta}(k) M(\mathrm{~d} \theta)$. Let $X_{\theta}^{j}(\tau)$ and $\Theta^{j}, j \in \mathbb{N}, \tau>0$, be random variables on the same probability space distributed as $X_{\theta}(\tau)$ and $\Theta$, respectively, all independent, and define the approximate process

$$
S_{\tau}(t)=\sum_{j=1}^{\lfloor t / \tau\rfloor} X_{\Theta^{j}}^{j}(\tau)
$$

We are ready to state the first theorem.

Theorem 1. Let $X$ be a stochastic process in $\mathbb{R}^{d}$ with stationary, independent increments and a Lévy measure with Lévy decomposition $\left(\phi_{\theta}, M\right)$. If

$$
\int_{\Omega_{\theta}} \mid \exp \left(\tau\left|\psi_{\theta}(k)\right|\right) M(\mathrm{~d} \theta)<\infty
$$

for some $\tau>0$ and all $k \in \mathbb{R}^{d}$, then $S_{\tau} \rightarrow X$ in the Skorokhod topology as $\tau \rightarrow 0+$.

Proof. By design, $S_{\tau}$ has independent increments. According to [9, Corollary VII.4.43] all we have to show is that the characteristic function of $S_{\tau}(t)$ converges to the characteristic function $\exp (t \psi(k))$ of $X(t)$ uniformly on compact intervals in $t$ for all $k \in \mathbb{R}^{d}$. 
Conditioning on $\Theta$, the characteristic function of $S_{\tau}(t)$ is given by

$$
\mathrm{E}\left[\mathrm{e}^{-\mathrm{i}\left\langle k, S_{\tau}(t)\right\rangle}\right]=\left(\int_{\Omega_{\theta}} \exp \left(\tau \psi_{\theta}(k)\right) M(\mathrm{~d} \theta)\right)^{\lfloor t / \tau\rfloor} .
$$

Using the facts that $a^{n}-b^{n}=(a-b) \sum_{j=0}^{n-1} a^{j} b^{n-j-1}$ and $|\exp (\tau \psi(k))| \leq 1$, we see that

$$
\begin{aligned}
\left|\exp (t \psi(k))-\mathrm{E}\left[\mathrm{e}^{-\mathrm{i}\left\langle k, S_{\tau}(t)\right\rangle}\right]\right| & \\
= & \left|\exp (t \psi(k))-\exp (\tau \psi(k))^{\lfloor t / \tau\rfloor}+\exp (\tau \psi(k))^{\lfloor t / \tau\rfloor}-\mathrm{E}\left[\mathrm{e}^{-\mathrm{i}\left\langle k, S_{\tau}(t)\right\rangle}\right]\right| \\
\leq & \left.\mid \exp (t \psi(k))-\exp \left(\tau \mid \frac{t}{\tau}\right\rfloor \psi(k)\right) \mid \\
& +\left\lfloor\frac{t}{\tau}\right\rfloor\left|\exp (\tau \psi(k))-\int_{\Omega_{\theta}} \exp \left(\tau \psi_{\theta}(k)\right) M(\mathrm{~d} \theta)\right| .
\end{aligned}
$$

Using Taylor's expansion, the last term may be bounded as

$$
\begin{aligned}
& \left\lfloor\frac{t}{\tau}\right\rfloor\left|\exp (\tau \psi(k))-\int_{\Omega_{\theta}} \exp \left(\tau \psi_{\theta}(k)\right) M(\mathrm{~d} \theta)\right| \\
& \quad=\left\lfloor\frac{t}{\tau}\right\rfloor\left|\sum_{j=2}^{\infty} \frac{(\tau \psi(k))^{j}}{j !}-\int_{\Omega_{\theta}} \sum_{j=2}^{\infty} \frac{\left(\tau \psi_{\theta}(k)\right)^{j}}{j !} M(\mathrm{~d} \theta)\right| \\
& \quad \leq \tau^{2}\left\lfloor\frac{t}{\tau}\right\rfloor|\psi(k)|^{2} \exp (\tau|\psi(k)|)+\tau^{2}\left\lfloor\frac{t}{\tau}\right\rfloor \int_{\Omega_{\theta}}\left|\psi_{\theta}(k)\right|^{2} \exp \left(\tau\left|\psi_{\theta}(k)\right|\right) M(\mathrm{~d} \theta) .
\end{aligned}
$$

It follows from our assumption on $\psi_{\theta}$ that the integral is bounded for fixed $k \in \mathbb{R}^{d}$ and small enough $\tau$, and, hence, (2) converges to 0 uniformly in $t$ on compact sets. Since the first term in (1) converges to 0 uniformly on compacta as well, the proof is complete.

Example 1. Let $X(t)$ be an operator stable process. Using the Jurek coordinate system [10], the log-characteristic function can be written as

$$
\psi(k)=\int_{\|\theta\|=1} \int_{0}^{\infty}\left(\mathrm{e}^{-\mathrm{i}\left\langle k, r^{E} \theta\right\rangle}-1+\frac{\mathrm{i}\left\langle k, r^{E} \theta\right\rangle}{1+\left\|r^{E} \theta\right\|^{2}}\right) \frac{c}{r^{2}} \mathrm{~d} r M(\mathrm{~d} \theta)
$$

for some scaling matrix $E$ with eigenvalues whose real part is larger than $\frac{1}{2}$. By Theorem 1 , this process can be approximated by generating steps via randomly (according to $M$ ) choosing a direction $\Theta$ and generating a random variable with log-characteristic function

$$
\tau \psi_{\Theta}(k)=\tau \int_{0}^{\infty}\left(\mathrm{e}^{-\mathrm{i}\left\langle k, r^{E} \Theta\right\rangle}-1+\frac{\mathrm{i}\left\langle k, r^{E} \Theta\right\rangle}{1+\left\|r^{E} \Theta\right\|^{2}}\right) \frac{c}{r^{2}} \mathrm{~d} r .
$$

Example 2. Let $X(t)$ be a tempered operator stable process with uniform scaling, i.e. its logcharacteristic function can be written (see [4]) as

$$
\begin{aligned}
\psi(k) & =\int_{\|\theta\|=1} \int_{0}^{\infty}\left(\mathrm{e}^{-\mathrm{i}\langle k, \theta\rangle r}-1+\mathrm{i}\langle k, \theta\rangle r\right) \frac{\mathrm{e}^{-r \rho(\theta)}}{r^{\alpha+1}} \mathrm{~d} r M(\mathrm{~d} \theta) \\
& =c \int_{\|\theta\|=1}\left[(\langle\mathrm{i} k, \theta\rangle+\rho(\theta))^{\alpha}-\rho(\theta)^{\alpha}-\alpha\langle\mathrm{i} k, \theta\rangle \rho(\theta)^{\alpha-1}\right] M(\mathrm{~d} \theta)
\end{aligned}
$$

for some $1<\alpha<2, c>0$, and exponential taper $\rho$, that is, a bounded measurable function $\rho: S^{d-1} \rightarrow \mathbb{R}^{+}$, where $S^{d-1}=\left\{\theta \in \mathbb{R}^{d}:\|\theta\|=1\right\}$. By Theorem 1, this process can 
be approximated by generating steps via randomly choosing a direction $\Theta$ (according to $M$ ) and generating a one-dimensional exponentially tempered stable random variable $Y_{\Theta}$ with tempering coefficient $\rho(\Theta)$ and time scale $\tau$ (see [1] for an efficient algorithm to generate tempered stable variables with given time scale and tempering coefficient), and letting $X_{\Theta}=$ $\Theta Y_{\Theta}$.

\section{Rate of convergence for tempered stable processes}

In the case of most tempered stable processes we can go further and actually provide a rate at which the densities of the processes converge. We are going to show that the characteristic functions converge uniformly at a rate of $o\left(\log ^{2} n / n\right)$, which translates into an $L^{2}$-multiplier convergence of the densities or uniform convergence if an arbitrarily small normal random variable is added to the process.

Definition 2. Let $\rho(\theta) \geq 0$ be a bounded measurable function on $S^{d-1}, 1<\alpha<2$, and $a>0$. Let

$$
A_{\theta}: k \mapsto a(\mathrm{i}\langle k, \theta\rangle+\rho(\theta))^{\alpha}-a \rho(\theta)^{\alpha}-\alpha a \rho(\theta)^{\alpha-1} \mathrm{i}\langle k, \theta\rangle
$$

be the tempered fractional derivative symbol in the direction $\theta$. If $\rho(\theta)=0$ then we call $A_{\theta}: k \mapsto a\langle\mathrm{i} k, \theta\rangle^{\alpha}$ the fractional derivative symbol in the direction of $\theta$.

We call $\rho$ the taper and the extended real-valued function

$$
F_{C}: \theta \mapsto \lim _{\varepsilon \rightarrow 0+}\left(\frac{\operatorname{essup}_{\|\theta\|=1} \rho(\theta)+\varepsilon}{\rho(\theta)+\varepsilon}\right)^{2-\alpha}
$$

the normalized fractional content of the taper at $\theta$.

For a probability measure $M$ on $S^{d-1}$, define the (tempered) fractional derivative symbol to be

$$
A: k \mapsto \int_{\|\theta\|=1} A_{\theta}(k) M(\mathrm{~d} \theta) .
$$

We say that a tempered fractional derivative symbol is full if

$$
\lambda_{M}=\min _{\|\eta\|=1} \int_{\|\theta\|=1}\langle\eta, \theta\rangle^{2} M(\mathrm{~d} \theta)>0 .
$$

It is easy to show that $\lambda_{M}$ is the smallest eigenvalue of the covariance matrix of $M$ viewed as a measure on $\mathbb{R}^{d}$, and is 0 if and only if $M$ is supported on a subspace.

Our main theorem is as follows.

Theorem 2. Let $A$ be a full, tempered fractional derivative symbol, and assume that $F_{C} \in$ $L^{2}\left(S^{d-1}, M(\mathrm{~d} \theta)\right)$. Then there exists a $C \geq 0$ such that

$$
\left|\left(\int_{\|\theta\|=1} \mathrm{e}^{t A_{\theta}(k) / n} M(\mathrm{~d} \theta)\right)^{n}-\mathrm{e}^{t A(k)}\right| \leq C \frac{1+\log ^{2} n}{n}
$$

for all $k \in \mathbb{R}^{d}, n \in \mathbb{N}$, and $t \geq 0$.

Proof. See Section 4. 
Let $\psi \in L^{\infty}\left(\mathbb{R}^{d}\right)$, and let $S\left(\mathbb{R}^{d}\right)$ denote the space of Schwartz functions. We call $\psi$ an $L^{p}$-Fourier multiplier, $1 \leq p<\infty$, if the map

$$
S\left(\mathbb{R}^{n}\right) \ni f \mapsto T_{\psi} f:=\mathcal{F}^{-1}(\psi \mathcal{F}(f))
$$

extends to a bounded linear operator on $L^{p}\left(\mathbb{R}^{d}\right)$, where the Fourier transform of $f$ is denoted by $\mathcal{F}(f)(k)=\int_{\mathbb{R}^{d}} \mathrm{e}^{-\mathrm{i}\langle k, x\rangle} f(x) \mathrm{d} x$. It is well known that, for a bounded Borel measure $\mu$ on $\mathbb{R}^{d}$, its Fourier transform $\hat{\mu}(k)=\int_{\mathbb{R}^{d}} \mathrm{e}^{-\mathrm{i}\langle k, x\rangle} \mu(\mathrm{d} x)$ is an $L^{p}$-Fourier multiplier and

$$
T_{\hat{\mu}} f=\mu * f .
$$

The Fourier multiplier $p$-norm of $\mu$ is defined as

$$
\|\mu\|_{\mathcal{M}_{p}\left(\mathbb{R}^{d}\right)}:=\sup _{\|f\|_{L^{p}\left(\mathbb{R}^{d}\right)}=1}\|\mu * f\|_{L^{p}\left(\mathbb{R}^{d}\right)}=\left\|T_{\hat{\mu}}\right\|_{\mathcal{B}\left(L^{p}\left(\mathbb{R}^{d}\right)\right)},
$$

where ' $*$ ' denotes the convolution and $\|\cdot\|_{\mathcal{B}\left(L^{p}\left(\mathbb{R}^{d}\right)\right)}$ denotes the operator norm on $L^{p}\left(\mathbb{R}^{d}\right)$.

Let $\mu_{t}$ and $v_{t}$ be probability measures with Fourier transforms

$$
\hat{\mu}_{t}(k)=\int_{\|\theta\|=1} \mathrm{e}^{t A_{\theta}(k)} M(\mathrm{~d} \theta)
$$

and

$$
\hat{v}_{t}(k)=\exp \left(t \int_{\|\theta\|=1} A_{\theta}(k) M(\mathrm{~d} \theta)\right),
$$

and let $\mu^{n *}$ denote the $n$th convolution power of a measure $\mu$.

Corollary 1. Let $A$ be a full, tempered fractional derivative symbol, and assume that $F_{C} \in$ $L^{2}\left(S^{d-1}\right)$. Then, for all $\varepsilon>0$, there exists a $C \geq 0$ such that

$$
\left\|\mu_{t / n}^{n *}-v_{t}\right\|_{\mathcal{M}_{2}\left(\mathbb{R}^{d}\right)} \leq C \frac{1+\log ^{2} n}{n}
$$

for all $n \in \mathbb{N}$ and $t \geq 0$.

Proof. Since, for a bounded Borel measure $\mu$, we have $\|\mu\|_{\mathcal{M}_{2}\left(\mathbb{R}^{d}\right)}=\sup _{k \in \mathbb{R}^{d}}|\hat{\mu}(k)|$, the statement follows from Theorem 2.

The next corollary translates $L^{2}$-multiplier convergence into uniform convergence in the presence of a small perturbation $\delta N(0,1)$, where $N(0,1)$ is the multivariate standard normal random variable.

Corollary 2. Let X be a tempered stable process with characteristic function

$$
\mathrm{E}\left[\mathrm{e}^{-\mathrm{i}\langle k, X(t)\rangle}\right]=\exp (t A(k))
$$

where $A$ is a full, tempered fractional derivative symbol with $F_{C} \in L^{2}\left(S^{d-1}\right)$. For $j \in \mathbb{N}$, $\tau>0$, and $\|\theta\|=1$, let $\Theta_{j}$ and $Y_{j, \theta}(\tau)$ be random variables on the same probability space, all independent, with $\Theta_{j}$ distributed as $\operatorname{Pr}\left\{\Theta_{j} \in \Omega\right\}=M(\Omega)$ and the distribution of $Y_{j, \theta}$ satisfying

$$
\mathrm{E}\left[\mathrm{e}^{-\mathrm{i}\left\langle k, Y_{j, \theta}(\tau)\right\rangle}\right]=\exp \left(\tau A_{\theta}(k)\right) .
$$

Then, for all $\delta>0$, there exists a $C>0$ such that the marginal densities of the approximate process $\delta N(0,1)+\sum_{j=1}^{\lfloor t / \tau\rfloor} \Theta_{j} Y_{j, \Theta_{j}}(\tau)$ converge uniformly in $x \in \mathbb{R}^{d}$ and $t \geq 0$ at a rate of $C \tau\left(1+\log ^{2}(1 / \tau)\right)$ to the marginal densities of $\delta N(0,1)+X(t)$ as $\tau \rightarrow 0+$. 


\section{Proof. See Section 4.}

The next theorem is important when it is used in conjunction with Corollary 1, as it allows the mixing of operators with different $\alpha$ s or tempered and untempered $(\rho \equiv 0)$ operators.

Theorem 3. Let $A_{j} \in C\left(\mathbb{R}^{d}\right), j=1, \ldots, m$, be sectorial, i.e. assume that there is ac $>0$ such that $\operatorname{Re}\left(A_{j}(k)\right) \leq-c\left|A_{j}(k)\right|$ for $k \in \mathbb{R}^{d}$ and all $j=1, \ldots, m$, and let $\mu_{t}$ and $v_{t}$ be probability measures with Fourier transforms $\hat{\mu}_{t}(k)=\sum \lambda_{j} \mathrm{e}^{t A_{j}(k)}$ and $\hat{v}_{t}(k)=\exp \left(t \sum \lambda_{j} A_{j}(k)\right)$. Then, for each collection of $0<\lambda_{j}<1$ with $\sum \lambda_{j}=1$, there exists $a>0$ such that

$$
\left\|\mu_{t / n}^{n *}-v_{t}\right\|_{\mathcal{M}_{2}\left(\mathbb{R}^{d}\right)} \leq C \frac{1+\log ^{2} n}{n}
$$

for all $n \in \mathbb{N}$ and $t \geq 0$.

Proof. As in the proof of Corollary 1, we use the fact that

$$
\left\|\mu_{t / n}^{n *}-v_{t}\right\|_{\mathcal{M}_{2}\left(\mathbb{R}^{d}\right)}=\left\|\left(\sum \lambda_{j} \mathrm{e}^{t A_{j} / n}\right)^{n}-\exp \left(t \sum \lambda_{j} A_{j}\right)\right\|_{L^{\infty}\left(\mathbb{R}^{d}\right)} .
$$

Without loss of generality, assume that $0<\lambda_{1} \leq \lambda_{j}$, which implies that $\lambda_{1} \leq 1 / m$. We divide the proof into two cases.

Case 1. Assume that $k \in \mathbb{R}^{d}$ is such that

$$
\sum \lambda_{j}\left|\frac{t}{n} A_{j}(k)\right| \leq \frac{2 m \log n}{c \lambda_{1} n} .
$$

Then $t\left|A_{j}(k)\right| / n \leq 2 m \log n /\left(\lambda_{1}^{2} c n\right)$ and, by the binomial formula,

$$
\begin{aligned}
\mid\left(\sum\right. & \left.\lambda_{j} \mathrm{e}^{t A_{j}(k) / n}\right)^{n}-\exp \left(t \sum \lambda_{j} A_{j}(k)\right) \mid \\
\leq & n\left|\left(\sum \lambda_{j} \mathrm{e}^{t A_{j}(k) / n}\right)-\exp \left(\sum \lambda_{j} \frac{t}{n} A_{j}(k)\right)\right| \\
& \leq n\left(\sum \lambda_{j}\left|\frac{t}{n} A_{j}(k)\right|^{2} \mathrm{e}^{t\left|A_{j}(k)\right| / n}+\left|\sum \lambda_{j} \frac{t}{n} A_{j}(k)\right|^{2} \exp \left(\sum \lambda_{j} \frac{t}{n}\left|A_{j}(k)\right|\right)\right) \\
& \leq n C\left(\frac{\log n}{n}\right)^{2} \\
& =\frac{C \log ^{2} n}{n} .
\end{aligned}
$$

Case 2. Assume that $k \in \mathbb{R}^{d}$ is such that

$$
\sum \lambda_{j}\left|\frac{t}{n} A_{j}(k)\right| \geq \frac{2 m \log n}{c \lambda_{1} n} .
$$

Then there exists $j_{1}$ such that $\lambda_{j_{1}}\left|t A_{j_{1}}(k) / n\right| \geq 2 \log n /\left(c \lambda_{1} n\right)$ and, hence,

$$
\begin{aligned}
\left|\sum \lambda_{j} \mathrm{e}^{t A_{j}(k) / n}\right| & \leq \lambda_{j_{1}} \mathrm{e}^{-c\left|t A_{j_{1}}(k) / n\right|}+\left(1-\lambda_{j_{1}}\right) \\
& \leq \lambda_{j_{1}} \mathrm{e}^{-2 c \log n / c \lambda_{1} n}+\left(1-\lambda_{j_{1}}\right) \\
& =\lambda_{j_{1}} \mathrm{e}^{-2 \log n / \lambda_{1} n}+\left(1-\lambda_{j_{1}}\right) .
\end{aligned}
$$


It can be seen by differentiating with respect to $x$ that if $0 \leq x \leq \ln 2$ then $\lambda \mathrm{e}^{-x}+(1-\lambda) \leq$ $\mathrm{e}^{-\lambda x / 2}$ and, hence, as $\lambda_{1} \leq \lambda_{j_{1}}$,

$$
\lambda_{j_{1}} \mathrm{e}^{-2 \log n / \lambda n}+\left(1-\lambda_{j_{1}}\right) \leq \mathrm{e}^{-\log n / n}
$$

Hence,

$$
\left|\left(\sum \lambda_{j} \mathrm{e}^{t A_{j}(k) / n}\right)^{n}-\exp \left(t \sum \lambda_{j} A_{j}(k)\right)\right| \leq \mathrm{e}^{-\log n}+\mathrm{e}^{-2 m \log n / \lambda_{1}} \leq \frac{C}{n} .
$$

By cases 1 and 2, there exists a $C$ such that

$$
\left\|\left(\sum \lambda_{j} \mathrm{e}^{t A_{j} / n}\right)^{n}-\exp \left(t \sum \lambda_{j} A_{j}\right)\right\|_{L^{\infty}\left(\mathbb{R}^{d}\right)} \leq \frac{C\left(1+\log ^{2} n\right)}{n}, \quad n \in \mathbb{N}, t \geq 0,
$$

which completes the proof in view of (4).

The next corollary allows us to approximate each $\mathrm{e}^{t A_{j} / n}$ with its polar approximation.

Corollary 3. Let $A_{j}=\int_{\|\theta\|=1} A_{j, \theta} M_{j}(\mathrm{~d} \theta), j=1, \ldots, m$, be tempered fractional derivative operators each satisfying the conditions of Theorem 2 , and let $\mu_{t}$ and $v_{t}$ be probability measures with Fourier transforms $\hat{\mu}_{t}(k)=\sum \lambda_{j} \int_{\|\theta\|=1} \mathrm{e}^{t A_{j, \theta}(k)} M_{j}(\mathrm{~d} \theta)$ and $\hat{v}_{t}(k)=\exp \left(t \sum \lambda_{j} A_{j}(k)\right)$. Then, for each collection of $0<\lambda_{j}<1$ with $\sum \lambda_{j}=1$, there exists a $C>0$ such that

$$
\left\|\mu_{t / n}^{n *}-v_{t}\right\|_{\mathcal{M}_{2}\left(\mathbb{R}^{d}\right)} \leq C \frac{1+\log ^{2} n}{n}
$$

for all $n \in \mathbb{N}$ and $t \geq 0$.

Proof. The proof follows by a straightforward combination of the proofs of Theorems 2 and 3 using the case in which

$$
\sum \lambda_{j} \int_{\|\theta\|=1}\left|\frac{t}{n} A_{j, \theta}(k)\right| M_{j}(\mathrm{~d} \theta) \leq \frac{2 m}{\lambda_{1}} \frac{\log n}{\tilde{c} n}
$$

or not, with $\tilde{c}=c_{S} c_{3}$, where $\lambda_{1}, c_{S}$, and $c_{3}$ are respectively the smallest of the $\lambda_{i}$, the constants in property 1 of Proposition 1 below, and the constants $c$ from Proposition 2 below.

We complete this section with an example highlighting the intended usage of Corollaries 1 , 2 , and 3 .

Example 3. Consider a process on $\mathbb{R}^{2}$ that has $\psi(k)=(1 / 2 \pi) \int_{0}^{2 \pi} A_{\theta}(k) \mathrm{d} \theta$ as its log-characteristic function, where

$$
A_{\theta}(k)= \begin{cases}(\langle\mathrm{i} k, \vec{\theta}\rangle+\sin (\theta))^{1.6}-\sin ^{1.6}(\theta)-(1.6) \sin ^{0.6}(\theta)\langle\mathrm{i} k, \vec{\theta}\rangle, & 0 \leq \theta \leq \pi, \\ \langle\mathrm{i} k, \vec{\theta}\rangle^{1.2}, & \pi<\theta<3 \pi / 2, \\ (2 \pi-\theta)\langle\mathrm{i} k, \vec{\theta}\rangle^{1.8}, & 3 \pi / 2<\theta<2 \pi,\end{cases}
$$

and $\vec{\theta}=(\cos \theta, \sin \theta)$. In order to apply Corollary 3 , let $\lambda_{1}=\frac{1}{2}, \lambda_{2}=\lambda_{3}=\frac{1}{4}$,

$$
\begin{gathered}
M_{1}(\mathrm{~d} x)=\frac{1}{\pi} I_{[0, \pi]}(x) \mathrm{d} x, \quad M_{2}(\mathrm{~d} x)=\frac{2}{\pi} I_{[\pi, 3 \pi / 2]}(x) \mathrm{d} x, \\
\text { and } M_{3}(\mathrm{~d} x)=\frac{8}{\pi^{2}} I_{[3 \pi / 2,2 \pi]}(x)(2 \pi-x) \mathrm{d} x .
\end{gathered}
$$


So we can rewrite $\psi(k)$ as

$$
\psi(k)=\lambda_{1} \int_{0}^{2 \pi} A_{\theta}(k) M_{1}(\mathrm{~d} \theta)+\lambda_{2} \int_{0}^{2 \pi} A_{\theta}(k) M_{2}(\mathrm{~d} \theta)+\lambda_{3} \int_{0}^{2 \pi} \frac{\pi}{4}\langle\mathrm{i} k, \vec{\theta}\rangle^{1.8} M_{3}(\mathrm{~d} \theta) .
$$

By the developed theory, the process can be faithfully approximated by generating increments with step size $\tau$, where each increment is generated by first generating a uniformly distributed random variable $\tilde{\Theta}$ over $[0,2 \pi]$, letting $\Theta=\tilde{\Theta}$ for $\tilde{\Theta}<3 \pi / 2$ and $\Theta=2 \pi-\sqrt{\pi^{2}-\tilde{\Theta} \pi / 2}$ otherwise, and then generating a one-dimensional random variable $X_{\Theta}$ with characteristic function

$$
\mathrm{E}\left[\exp \left(-\mathrm{i} k X_{\Theta}\right)\right]=\exp \left(\tau \tilde{A}_{\Theta}(k)\right)
$$

where

$$
\tilde{A}_{\theta}(k)= \begin{cases}(\mathrm{i} k+\sin (\theta))^{1.6}-\sin ^{1.6}(\theta)-(1.6) \sin ^{0.6}(\theta) \mathrm{i} k, & 0 \leq \theta \leq \pi, \\ (\mathrm{i} k)^{1.2}, & \pi<\theta<3 \pi / 2, \\ \frac{\pi}{4}(\mathrm{i} k)^{1.8}, & 3 \pi / 2<\theta<2 \pi\end{cases}
$$

The one-dimensional variables can be generated (or approximated) using the methods in [1] and [6]. The increment is then given by $X_{\Theta} \vec{\Theta}$. In Figure 1 we plot a sample path over the time intervals $t<1, t<100$, and $t<1000$, generated with $\tau=1 / 1000$. In Figure 2 we plot the $\log$ density estimate at time $t=1$ taken from 1000000 sample paths generated with $\tau=\frac{1}{64}$. In Table 1 we show the absolute error decreasing between estimates for different $\tau$. As the error from one simulation to the next with $\tau=\frac{1}{64}$ is of the order of the error between simulations with decreased $\tau$, we stopped at $\tau=\frac{1}{64}$.
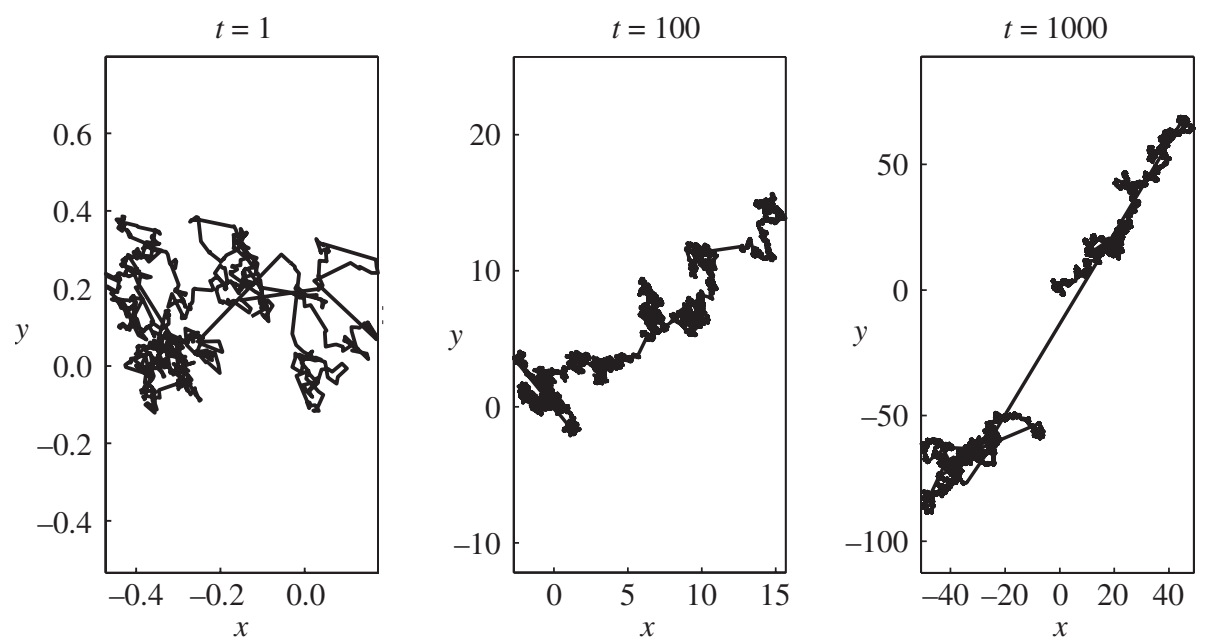

Figure 1: A simulated sample path of Example 3, generated with $\tau=0.001$. Note that relatively large upward jumps are present in the small and medium scales virtually disappear in the larger scale on the right as the probability of jumps larger than $x$ is less than $\exp (-x \sin (\theta))$ for $0<\theta<\pi$. 

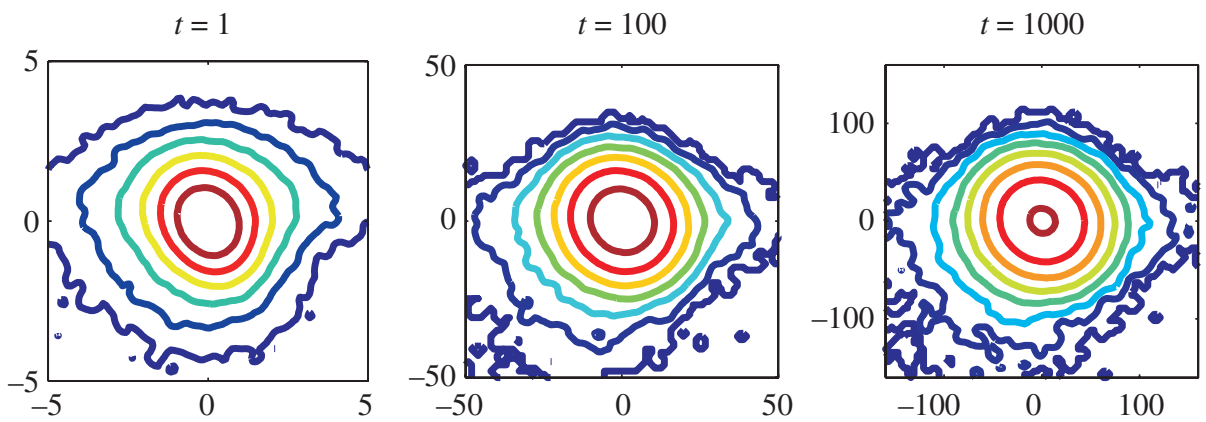

Figure 2: The estimated log densities of Example 3, generated with 1000000 sample paths and a small Gaussian smoothing. Level sets depict a decrease by 1.5 (in log space), starting innermost with $-3,-7.5$, and -9 respectively.

TABLE 1: Absolute error in Monte Carlo density estimates of 1000000 sample paths to $t=1$ for different time steps. At $\tau=\frac{1}{64}$ the error is between different realisations.

\begin{tabular}{lccccccc}
\hline$\tau$ & 1 & $\frac{1}{2}$ & $\frac{1}{4}$ & $\frac{1}{8}$ & $\frac{1}{16}$ & $\frac{1}{32}$ & $\frac{1}{64}$ \\
$\sup \left|f_{\tau}-f_{1 / 64}\right|$ & 0.107 & 0.072 & 0.045 & 0.020 & 0.008 & 0.004 & 0.003 \\
\hline
\end{tabular}

\section{Proofs of Theorem 2 and Corollary 2}

Proposition 1. Let A be a tempered fractional derivative symbol. Let $u=\langle k, \theta\rangle$, where $k \in \mathbb{R}^{d}$ and $\theta \in S^{d-1}$. Then the following properties hold.

1. There exists a constant $c_{S}>0$ such that

$$
\operatorname{Re}\left(A_{\theta}(k)\right) \leq-c_{S}\left|A_{\theta}(k)\right|
$$

for all $k$ and $\theta$, i.e. $A_{\theta}$ and $A$ are sectorial.

2. There exist constants $c_{L}, c_{U}>0$ such that

$$
c_{L} \min \left\{\frac{u^{2}}{\rho(\theta)^{2-\alpha}},|u|^{\alpha}\right\} \leq\left|A_{\theta}(k)\right| \leq c_{U} \min \left\{\frac{u^{2}}{\rho(\theta)^{2-\alpha}},|u|^{\alpha}\right\}
$$

for all $k$ and $\theta$ with $\rho(\theta)>0$; if $\rho(\theta)=0, c_{L}|u|^{\alpha} \leq\left|A_{\theta}(k)\right| \leq c_{U}|u|^{\alpha}$.

Proof. Note that without loss of generality we can set $\rho(\theta)=a=1$ as the general case follows by replacing $u$ (or, equivalently, $k$ ) with $u / \rho(\theta)$ and multiplying $A_{\theta}$ by $a \rho(\theta)^{\alpha}$. As

$$
(\mathrm{i} u+1)^{\alpha}-1-\alpha \mathrm{i} u=(\mathrm{i} u)^{\alpha}+o\left(|u|^{\alpha}\right)
$$

as $u \rightarrow \infty$ and, by the Taylor expansion,

$$
(\mathrm{i} u+1)^{\alpha}-1-\alpha \mathrm{i} u=-\frac{\alpha(\alpha-1)}{2} u^{2}+o\left(u^{2}\right)
$$

as $u \rightarrow 0$, the inequalities follow once we establish that $\left|\operatorname{Re}\left(A_{\theta}\right)\right|$ and $\left|\operatorname{Im}\left(A_{\theta}\right)\right|$ with $\rho(\theta)=1$ are continuous, increasing functions of $|u|$. 
First we show that $\operatorname{Re}\left((\mathrm{i} u+1)^{\alpha}-1\right)$ is decreasing for $u>0$; it is clearly continuous and differentiable. To that end, let $\phi=\arctan u$. Then $|i u+1|=\sec \phi$ and

$$
f(\phi)=\operatorname{Re}\left((\mathrm{i} \tan \phi+1)^{\alpha}-1\right)=\cos \alpha \phi \sec ^{\alpha} \phi-1 .
$$

Its derivative is given by

$$
\begin{aligned}
\frac{\mathrm{d}}{\mathrm{d} \phi} f(\phi) & =-\alpha \sin (\alpha \phi) \sec ^{\alpha}(\phi)+\cos (\alpha \phi) \alpha \sec ^{\alpha-1}(\phi) \sec (\phi) \tan (\phi) \\
& =-\alpha \sec ^{\alpha+1}(\phi)(\sin (\alpha \phi) \cos (\phi)-\cos (\alpha \phi) \sin (\phi)) \\
& =-\alpha \sec ^{\alpha+1}(\phi) \sin (\alpha \phi-\phi) \\
& <0
\end{aligned}
$$

for $\phi>0$ and positive for $\phi<0$. Hence, $f$ is decreasing for positive $\phi$ or $u$ and increasing for negative $u$, and since $f(0)=0,|f|$ is increasing for increasing $|\phi|$ or $|u|$.

Similarly, we show that $\operatorname{Im}\left((\mathrm{i} u+1)^{\alpha}-\alpha \mathrm{i} u\right)$ is increasing. Again, let $\phi=\arctan u$ and

$$
f(\phi)=\operatorname{Im}\left((\mathrm{i} \tan \phi+1)^{\alpha}-\alpha \mathrm{i} \tan \phi\right)=\sin \alpha \phi \sec ^{\alpha} \phi-\alpha \tan \phi .
$$

Then

$$
\begin{aligned}
\frac{\mathrm{d}}{\mathrm{d} \phi} f(\phi) & =\alpha \cos (\alpha \phi) \sec ^{\alpha}(\phi)+\sin (\alpha \phi) \alpha \sec ^{\alpha-1}(\phi) \sec (\phi) \tan (\phi)-\alpha \sec ^{2}(\phi) \\
& =\alpha \sec ^{\alpha+1}(\phi)(\cos (\alpha \phi) \cos (\phi)+\sin (\alpha \phi) \sin (\phi))-\alpha \sec ^{2} \phi \\
& =\alpha \sec ^{\alpha+1}(\phi) \cos (\alpha \phi-\phi)-\alpha \sec ^{2}(\phi) \\
& =\sec ^{2}(\phi)\left(\alpha \sec ^{\alpha-1}(\phi) \cos ((\alpha-1) \phi)-\alpha\right)
\end{aligned}
$$

At $\phi=0$, we have $\mathrm{d} f(\phi) / \mathrm{d} \phi=0$. The last factor in (7) is similar to (5) and its derivative, given by

$$
-\alpha(\alpha-1) \sec ^{\alpha}(\phi) \sin ((\alpha-2) \phi)>0
$$

for $\phi>0$ and negative for $\phi<0$, is computed similarly to (6). Hence, $\mathrm{d} f(\phi) / \mathrm{d} \phi>0$ for all $\phi \neq 0$, and since $f(0)=0,|f|$ is increasing for increasing $|u|$.

Lemma 1. Let $F_{C} \in L^{1}\left(S^{d-1}, M(\mathrm{~d} \theta)\right)$ and $\varepsilon>0$. Then there exists a constant $c>0$ such that

$$
\int_{\|\theta\|=1}\left|A_{\theta}(k)\right| M(\mathrm{~d} \theta) \geq \varepsilon
$$

implies that if $\rho_{\max }:=\operatorname{essup}_{\|\theta\|=1} \rho^{2-\alpha}(\theta)>0$,

$$
\min \left\{\|k\|^{2} \frac{\left\|F_{C}\right\|_{1}}{\rho_{\max }},\|k\|^{\alpha}\right\}>c \varepsilon ;
$$

otherwise, $\|k\|^{\alpha}>c \varepsilon$. 
Proof. Assume that $\rho_{\max }>0$. As $F_{C} \in L^{1}\left(S^{d-1}\right)$, the set for which $\rho(\theta)=0$ is a null set. By Proposition 1,

$$
\begin{aligned}
\int_{\|\theta\|=1}\left|A_{\theta}(k)\right| M(\mathrm{~d} \theta) & \leq c_{U} \int_{\|\theta\|=1} \min \left\{\left|\frac{\langle k, \theta\rangle^{2}}{\rho(\theta)^{2-\alpha}}\right|,|\langle k, \theta\rangle|^{\alpha}\right\} M(\mathrm{~d} \theta) \\
& \leq c_{U} \min \left\{\int_{\|\theta\|=1}\left|\frac{\langle k, \theta\rangle^{2}}{\rho(\theta)^{2-\alpha}}\right| M(\mathrm{~d} \theta), \int_{\|\theta\|=1}|\langle k, \theta\rangle|^{\alpha} M(\mathrm{~d} \theta)\right\} \\
& \leq c_{U} \min \left\{\|k\|^{2} \frac{\left\|F_{C}\right\|_{1}}{\rho_{\max }},\|k\|^{\alpha}\right\} .
\end{aligned}
$$

Hence

$$
\min \left\{\|k\|^{2} \frac{\left\|F_{C}\right\|_{1}}{\rho_{\max }},\|k\|^{\alpha}\right\} \geq \frac{\varepsilon}{c_{U}} .
$$

In the case where $\rho_{\max }=0,\left|A_{\theta}(k)\right|=a|\langle k, \theta\rangle|^{\alpha}$. Similarly, we then obtain $\|k\|^{\alpha} \geq \varepsilon / c_{U}$.

Lemma 2. There exists a $c>0$ such that if $\rho_{\max }:=\operatorname{essup}_{\|\theta\|=1} \rho(\theta)^{2-\alpha}>0$ then

$$
\left|A_{\theta}(k)\right| \geq c \min \left\{\frac{\|k\|^{2}}{\rho_{\max }},\|k\|^{\alpha}\right\} \frac{\langle k, \theta\rangle^{2}}{\|k\|^{2}} ;
$$

otherwise, $\left|A_{\theta}(k)\right| \geq c\|k\|^{\alpha-2}\langle k, \theta\rangle^{2}$.

Proof. If $\rho(\theta)=0$ then $\left|A_{\theta}(k)\right|=a|\langle k, \theta\rangle|^{\alpha} \geq a\|k\|^{\alpha}\langle k, \theta\rangle^{2} /\|k\|^{2}$. If $\rho(\theta)>0$, by Proposition 1 we have

$$
\begin{aligned}
\left|A_{\theta}(k)\right| & \geq c_{L} \min \left\{\left|\frac{\langle k, \theta\rangle^{2}}{\rho(\theta)^{2-\alpha}}\right|,|\langle k, \theta\rangle|^{\alpha}\right\} \\
& \geq c_{L} \min \left\{\frac{|\langle k, \theta\rangle|^{2}}{\rho_{\max }},\|k\|^{\alpha} \mid\left\langle\frac{k}{\|k\|},\left.\theta\right|^{\alpha}\right\}\right. \\
& \geq c_{L} \min \left\{\frac{\|k\|^{2}}{\rho_{\max }}\left\langle\frac{k}{\|k\|}, \theta\right\rangle^{2},\|k\|^{\alpha}\left\langle\frac{k}{\|k\|}, \theta\right\rangle^{2}\right\} \\
& =c_{L} \min \left\{\frac{\|k\|^{2}}{\rho_{\max }},\|k\|^{\alpha}\right\} \frac{\langle k, \theta\rangle^{2}}{\|k\|^{2}} .
\end{aligned}
$$

Lemma 3. Let $M$ be a probability measure on $[0,1]$. Then, for $\mu \geq 0$,

$$
\int_{0}^{1} \mathrm{e}^{-\mu u} M(\mathrm{~d} u) \leq \exp \left(-\frac{\min (\mu, 1)}{2} \int_{0}^{1} u M(\mathrm{~d} u)\right) .
$$

Proof. Clearly, for $u \in[0,1], \mathrm{e}^{-\mu u} \leq 1-\left(1-\mathrm{e}^{-\mu}\right) u$. Hence,

$$
\int_{0}^{1} \mathrm{e}^{-\mu u} M(\mathrm{~d} u) \leq 1-\left(1-\mathrm{e}^{-\mu}\right) \int_{0}^{1} u M(\mathrm{~d} u) \leq \exp \left(-\left(1-\mathrm{e}^{-\mu}\right) \int_{0}^{1} u M(\mathrm{~d} u)\right) .
$$

The assertion follows from the fact that, for $0 \leq \mu \leq 1,1-\mathrm{e}^{-\mu} \geq \mu / 2$ and, for $\mu>1$, $1-\mathrm{e}^{-\mu} \geq \frac{1}{2}$ 
Proposition 2. Let $A$ be a full, tempered fractional derivative symbol. Assume that $F_{C} \in$ $L^{1}\left(S^{d-1}, M(\mathrm{~d} \theta)\right)$. Then there exist $0<c \leq 1$ and $d>0$ such that, for all $\varepsilon, t>0$ and $n \in \mathbb{N}$,

$$
\int_{\|\theta\|=1} \frac{t}{n}\left|A_{\theta}(k)\right| M(\mathrm{~d} \theta) \geq \varepsilon
$$

implies that

$$
\int_{\|\theta\|=1} \mathrm{e}^{-t\left|A_{\theta}(k)\right| / n} M(\mathrm{~d} \theta) \leq \mathrm{e}^{-\min \{c \varepsilon, d\}} .
$$

Proof. Combining Lemma 1 and Lemma 2, there exist $c_{L}, c_{U}>0$ such that

$$
\begin{aligned}
\left|A_{\theta}(k)\right| & \geq c_{L} \min \left\{\frac{\|k\|^{2}}{\rho_{\max }},\|k\|^{\alpha}\right\} \frac{\langle k, \theta\rangle^{2}}{\|k\|^{2}} \\
& \geq \frac{n}{t} \frac{\varepsilon c_{L}}{c_{U}} \min \left\{\frac{1}{\left\|F_{C}\right\|_{1}}, 1\right\} \frac{\langle k, \theta\rangle^{2}}{\|k\|^{2}} \\
& =\frac{n}{t} \frac{\varepsilon c_{L}}{c_{U}\left\|F_{C}\right\|_{1}} \frac{\langle k, \theta\rangle^{2}}{\|k\|^{2}} .
\end{aligned}
$$

Define a measure $M_{k}$ on $[0,1]$ via

$$
M_{k}(\Omega)=M\left(\left\{\theta: \frac{\langle k, \theta\rangle^{2}}{\|k\|^{2}} \in \Omega\right\}\right)
$$

for each measurable $\Omega \subset[0,1]$. Then, by Lemma 3 and inequality (8), there exists $0<c \leq 1$ such that

$$
\begin{aligned}
\int_{\|\theta\|=1} \mathrm{e}^{-t\left|A_{\theta}(k)\right| / n} M(\mathrm{~d} \theta) & \leq \int_{\|\theta\|=1} \exp \left(-\frac{t}{n} \frac{n}{t} \frac{\varepsilon c_{L}}{c_{U}\left\|F_{C}\right\|_{1}} \frac{\langle k, \theta\rangle^{2}}{\|k\|^{2}}\right) M(\mathrm{~d} \theta) \\
& \leq \int_{0}^{1} \exp \left(-\frac{\varepsilon c_{L}}{c_{U}\left\|F_{C}\right\|_{1}} u\right) M_{k}(\mathrm{~d} u) \\
& \leq \exp \left(-\frac{1}{2} \min \left\{\frac{\varepsilon c_{L}}{c_{U}\left\|F_{C}\right\|_{1}}, 1\right\} \int_{0}^{1} u M_{k}(\mathrm{~d} u)\right) \\
& \leq \exp \left(-\frac{1}{2} \min \left\{\frac{\varepsilon c_{L}}{c_{U}\left\|F_{C}\right\|_{1}}, 1\right\} \lambda_{M}\right) \\
& \leq \mathrm{e}^{-\min \{c \varepsilon, d\}} .
\end{aligned}
$$

Lemma 4. Let A be a full, tempered fractional derivative symbol. Then there exists a constant $c>0$ such that, for all $\varepsilon>0$,

$$
\int_{\|\theta\|=1}\left|A_{\theta}(k)\right| M(\mathrm{~d} \theta) \leq \varepsilon
$$

implies that

$$
\left|A_{\theta}(k)\right|<c \varepsilon F_{C}(\theta) .
$$

Proof. By Lemma 2, if $\rho_{\max }:=\operatorname{essup}_{\|\theta\|=1} \rho(\theta)^{2-\alpha}>0$ then

$$
\left|A_{\theta}(k)\right| \geq c_{L} \min \left\{\frac{\|k\|^{2}}{\rho_{\max }},\|k\|^{\alpha}\right\} \frac{\langle k, \theta\rangle^{2}}{\|k\|^{2}},
$$


and, hence,

$$
\varepsilon \geq \int_{\|\theta\|=1}\left|A_{\theta}(k)\right| M(\mathrm{~d} \theta) \geq c_{L} \min \left\{\frac{\|k\|^{2}}{\rho_{\max }},\|k\|^{\alpha}\right\} \lambda_{M} .
$$

Therefore, either $\|k\|^{2} \leq \varepsilon \rho_{\max } / \lambda_{M} c_{L}$ or $\|k\|^{\alpha} \leq \varepsilon / \lambda_{M} c_{L}$. If $\rho_{\max }=0$, we clearly also have $\|k\|^{\alpha} \leq \varepsilon / \lambda_{M} c_{L}$. In the case where $\|k\|^{2} \leq \varepsilon \rho_{\max } / \lambda_{M} c_{L}$, this implies that

$$
\left|A_{\theta}(k)\right| \leq c_{U} \min \left\{\left|\frac{\langle k, \theta\rangle^{2}}{\rho(\theta)^{2-\alpha}}\right|,|\langle k, \theta\rangle|^{\alpha}\right\} \leq \frac{c_{U}}{\lambda_{M} c_{L}} F_{C}(\theta) \varepsilon ;
$$

in the case where $\|k\|^{\alpha} \leq \varepsilon / \lambda_{M} c_{L}$, this implies that

$$
\left|A_{\theta}(k)\right| \leq c_{U} \min \left\{\left|\frac{\langle k, \theta\rangle^{2}}{\rho(\theta)^{2-\alpha}}\right|,|\langle k, \theta\rangle|^{\alpha}\right\} \leq \frac{c_{U}}{\lambda_{M} c_{L}} \varepsilon .
$$

As $F_{C}(\theta) \geq 1$, the lemma is proven.

Proposition 3. Let $A$ be a full, tempered fractional derivative symbol, and assume that $F_{C} \in$ $L^{2}\left(S^{d-1}, M(\mathrm{~d} \theta)\right)$. Then there exists a constant $c>0$ such that, for all $0<\varepsilon \leq 1$ and all $n, t>0$,

implies that

$$
\frac{t}{n} \int_{\|\theta\|=1}\left|A_{\theta}(k)\right| M(\mathrm{~d} \theta) \leq \varepsilon
$$

$$
\left|\int_{\|\theta\|=1} \mathrm{e}^{t A_{\theta}(k) / n} M(\mathrm{~d} \theta)-\mathrm{e}^{t A(k) / n}\right| \leq c \varepsilon^{2} .
$$

Proof. Note that, by Lemma 4, there exists a constant $c=c_{U} / c_{L} \lambda_{M}$ such that

$$
\frac{t}{n}\left|A_{\theta}(k)\right| \leq c \varepsilon F_{C}(\theta) .
$$

Then, using the facts that $A(k)=\int_{\|\theta\|=1} A_{\theta}(k) M(\mathrm{~d} \theta)$ and $M$ is a probability measure,

$$
\begin{aligned}
& \mid \mathrm{e}^{t} \\
&=\left|\int_{\|\theta\|=1} \int_{0}^{1(k) / n}-\int_{\|\theta\|=1} \mathrm{e}^{t A_{\theta}(k) / n} M(\mathrm{~d} \theta)\right| \\
&=\mid \int_{\|\theta\|=1}\left[s\left(\frac{t}{n} A(k)-\frac{t}{n} A_{\theta}(k)\right) \exp \left(s \frac{t}{n} A(k)+(1-s) \frac{t}{n} A_{\theta}(k)\right) \mathrm{d} s M(\mathrm{~d} \theta) \mid\right. \\
&=\left|\int_{\|\theta\|=1} \int_{0}^{1} s\left(\frac{t}{n} A(k)-\frac{t}{n} A_{\theta}(k)\right) \exp \left(\frac{t}{n}\left(s A(k)+(1-s) A_{\theta}(k)\right)\right)\right|_{s=0}^{s=1} \exp \left(s \frac{t}{n} A(k)+(1-s) \frac{t}{n} A_{\theta}(k)\right) \mathrm{d} s M(\mathrm{~d} \theta) \mid \\
& \leq \frac{1}{2} \int_{\|\theta\|=1}\left|\frac{t}{n} A(k)-\frac{t}{n} A_{\theta}(k)\right|^{2} M(\mathrm{~d} \theta) \\
& \leq \frac{1}{2} \int_{\|\theta\|=1}\left|\frac{t}{n} A_{\theta}(k)\right|_{\theta}^{2} M(\mathrm{~d} \theta)+\frac{3}{2} \varepsilon^{2} \\
& \leq \frac{c^{2} \varepsilon^{2}}{2} \int_{\|\theta\|=1}\left|F_{C}(\theta)\right|^{2} M(\mathrm{~d} \theta)+\frac{3}{2} \varepsilon^{2} . \\
&
\end{aligned}
$$


Proof of Theorem 2. We divide the proof into two cases. First let

$$
\frac{t}{n} \int_{\|\theta\|=1}\left|A_{\theta}(k)\right| M(\mathrm{~d} \theta)>\frac{\log n}{\tilde{c} n},
$$

where $\tilde{c}=c_{S} c_{3}$. Here $c_{S}$ denotes the constant in property 1 of Proposition 1 and $c_{3} \leq 1$ is the constant $c$ from Proposition 2. Then, by property 1 of Proposition 1 ,

$$
\left|\exp \left(t \int_{\|\theta\|=1} A_{\theta}(k) M(\mathrm{~d} \theta)\right)\right| \leq \mathrm{e}^{-n c_{S} \log n / \tilde{c} n}=\frac{1}{n^{1 / c_{3}}} .
$$

Furthermore, by Proposition 2 we also have

$$
\left|\left(\int_{\|\theta\|=1} \mathrm{e}^{t A_{\theta}(k) / n} M(\mathrm{~d} \theta)\right)^{n}\right| \leq \mathrm{e}^{-c_{S} n \min \left\{c_{3} \log n / \tilde{c} n, d\right\}} .
$$

Hence, there exists a $C>0$ such that

$$
\left|\left(\int_{\|\theta\|=1} \mathrm{e}^{t A(k) / n}\right)^{n}-\mathrm{e}^{t A(k)}\right| \leq \frac{C}{n} .
$$

In the case

$$
\frac{t}{n} \int_{\|\theta\|=1}\left|A_{\theta}(k)\right| M(\mathrm{~d} \theta) \leq \frac{\log n}{\tilde{c} n},
$$

first note that, for $|a|,|b| \leq 1$,

$$
\left|a^{n}-b^{n}\right|=|a-b| \sum_{j=0}^{n-1}\left|a^{j} b^{n-1-j}\right| \leq n|a-b| .
$$

By Proposition 3, there exists a $c>0$ such that

$$
\begin{aligned}
\left|\left(\int_{\|\theta\|=1} \mathrm{e}^{t A_{\theta}(k) / n} M(\mathrm{~d} \theta)\right)^{n}-\left(\mathrm{e}^{t A(k) / n}\right)^{n}\right| & \leq n\left|\int_{\|\theta\|=1} \mathrm{e}^{t A_{\theta}(k) / n} M(\mathrm{~d} \theta)-\mathrm{e}^{t A(k) / n}\right| \\
& \leq n c\left(\frac{\log n}{\tilde{c} n}\right)^{2} \\
& =C \frac{\log ^{2} n}{n}
\end{aligned}
$$

By combining the two cases, it follows that there exists a $C>0$ such that (3) holds.

Proof of Corollary 2. Uniform convergence of the densities follows from the $L^{1}$-convergence of the characteristic function since $\|f\|_{\infty} \leq\|\hat{f}\|_{1} /(2 \pi)^{d}$, where

$$
\hat{f}: k \mapsto \int \mathrm{e}^{\mathrm{i}\langle k, x\rangle} f(x) \mathrm{d} x .
$$


Hence, we need to estimate

$$
\begin{aligned}
\left\|\mathrm{e}^{-\delta\|\cdot\|^{2} / 2}\left(\mathrm{e}^{t A(\cdot)}-\left(\int_{\|\theta\|=1} \mathrm{e}^{\tau A_{\theta}(\cdot)} M(\mathrm{~d} \theta)\right)^{\lfloor t / \tau\rfloor}\right)\right\|_{L^{1}\left(\mathbb{R}^{d}\right)} \\
\leq\left\|\mathrm{e}^{-\delta\|\cdot\|^{2} / 2}\left(\mathrm{e}^{t A(\cdot)}-\mathrm{e}^{\tau\lfloor t / \tau\rfloor A(\cdot)}\right)\right\|_{L^{1}\left(\mathbb{R}^{d}\right)} \\
\quad+\left\|\mathrm{e}^{-\delta\|\cdot\|^{2} / 2}\left(\mathrm{e}^{\tau\lfloor t / \tau\rfloor A(\cdot)}-\left(\int_{\|\theta\|=1} \mathrm{e}^{\tau A_{\theta}(\cdot)} M(\mathrm{~d} \theta)\right)^{\lfloor t / \tau\rfloor}\right)\right\|_{L^{1}\left(\mathbb{R}^{d}\right)} \\
=I_{1}+I_{2} .
\end{aligned}
$$

Using the fact that $\operatorname{Re} A(k) \leq 0$, we obtain

$$
I_{1} \leq\left\|\mathrm{e}^{-\delta\|\cdot\|^{2} / 2}\left|1-\mathrm{e}^{\tau A(\cdot)}\right|\right\|_{L^{1}\left(\mathbb{R}^{d}\right)} .
$$

Comparing real and imaginary parts we easily see that there exists a $c>0$ such that

$$
\left|1-\mathrm{e}^{\tau A(k)}\right| \leq c \tau(1+|A(k)|),
$$

and, hence, there exists a $C$ such that $I_{1} \leq C \tau$.

In order to estimate $I_{2}$, note that, by Proposition 1 , there exists a $c_{U}$ such that

$$
\max _{\|\theta\|=1}\left|A_{\theta}(k)\right| \leq c_{U}\|k\|^{\alpha}
$$

for all $k \in \mathbb{R}^{d}$. We divide the estimate into two parts.

Firstly, consider $t \leq 1$. Then using the same technique as in (9) and (10), we see that

$$
\begin{aligned}
\left|\mathrm{e}^{\tau\lfloor t / \tau\rfloor A(k)}-\left(\int_{\|\theta\|=1} \mathrm{e}^{\tau A_{\theta}(k)} M(\mathrm{~d} \theta)\right)^{\lfloor t / \tau\rfloor}\right| & \leq \frac{1}{2}\left\lfloor\frac{t}{\tau}\right\rfloor \tau^{2} \int_{\|\theta\|=1}\left|A_{\theta}(k)-A(k)\right|^{2} M(\mathrm{~d} \theta) \\
& \leq C \tau\|k\|^{2 \alpha},
\end{aligned}
$$

where the last inequality follows from Proposition 1 . Hence, for $t \leq 1$,

$$
I_{2} \leq C \tau\|\| \cdot\left\|^{2 \alpha} \mathrm{e}^{-\delta\|\cdot\|^{2} / 2}\right\|_{L^{1}\left(\mathbb{R}^{d}\right)} .
$$

For $t \geq 1$, apply Theorem 2 with $n=\lfloor t / \tau\rfloor$ to obtain

$$
\begin{aligned}
\left|\mathrm{e}^{\tau\lfloor t / \tau\rfloor A(k)}-\left(\int_{\|\theta\|=1} \mathrm{e}^{\tau A_{\theta}(k)} M(\mathrm{~d} \theta)\right)^{\lfloor t / \tau\rfloor}\right| & \leq C_{1} \frac{1+\log ^{2}\lfloor t / \tau\rfloor}{\lfloor t / \tau\rfloor} \\
& \leq C \tau\left(1+\log ^{2}\left(\frac{1}{\tau}\right)\right),
\end{aligned}
$$

and, hence, in this case

$$
I_{2} \leq C \tau\left(1+\log ^{2}\left(\frac{1}{\tau}\right)\right)\left\|\mathrm{e}^{-\delta\|\cdot\|^{2} / 2}\right\|_{L^{1}\left(\mathbb{R}^{d}\right)} .
$$

Thus, the marginal densities converge independently of $t$ at the prescribed rate. 


\section{Acknowledgement}

The authors thank the anonymous referee for his/her suggestions, which improved the quality of this paper.

\section{References}

[1] Baeumer, B. And Meerschaert, M. M. (2010). Tempered stable Lévy motion and transient super-diffusion. J. Comput. Appl. Math. 233, 2438-2448.

[2] Carr, P., Geman, H., Madan, D. B. and Yor, M. (2002). The fine structure of asset returns: an empirical investigation. J. Business 75, 305-332.

[3] Carr, P., Geman, H., Madan, D. B. and Yor, M. (2003). Stochastic volatility for Lévy processes. Math. Finance 13, 345-382.

[4] Cartea, A. and del Castillo-Negrete, D. (2007). Fluid limit of the continuous-time random walk with general Lévy jump distribution functions. Phys. Rev. E 76, 041105, 8pp.

[5] Chakrabarty, A. and Meerschaert, M. M. (2011). Tempered stable laws as random walk limits. Statist. Prob. Lett. 81, 989-997.

[6] Chambers, J. M., Mallows, C. L. And Stuck, B. W. (1976). A method for simulating stable random variables. J. Amer. Statist. Assoc. 71, 340-344.

[7] Cohen, S. AND Rosiński, J. (2007). Gaussian approximation of multivariate Lévy processes with applications to simulation of tempered stable processes. Bernoulli 13, 195-210.

[8] Cohen, S., Meerschaert, M. M. and Rosiński, J. (2010). Modeling and simulation with operator scaling. Stoch. Process. Appl. 120, 2390-2411.

[9] JACOD, J. AND Shiryaev, A. N. (2003). Limit Theorems for Stochastic Processes (Fundamental Principles Math. Sci. 288), 2nd edn. Springer, Berlin.

[10] Jurek, Z. J. And Mason, J. D. (1993). Operator-Limit Distributions in Probability Theory. John Wiley, New York.

[11] Koponen, I. (1995). Analytic approach to the problem of convergence of truncated Lévy flights towards the Gaussian stochastic process. Phys. Rev. E 52, 1197-1199.

[12] Meerschaert, M. M. And Scheffler, H.-P. (2001). Limit Distributions for Sums of Independent Random Vectors. John Wiley, New York.

[13] Meerschaert, M. M., Zhang, Y. and Baeumer, B. (2008). Tempered anomalous diffusion in heterogeneous systems. Geophys. Res. Lett. 35, 8-11.

[14] Novikov, E. A. (1994). Infinitely divisible distributions in turbulence. Phys. Rev. E 50, R3303-R3305.

[15] Rosiński, J. (2007). Tempering stable processes. Stoch. Process. Appl. 117, 677-707.

[16] Zhang, Y., Baeumer, B. And Reeves, D. M. (2010). A tempered multiscaling stable model to simulate transport in regional-scale fractured media. Geophys. Res. Lett. 37, L11405, 5pp.

[17] ZhANG, Y. et al. (2006). Random walk approximation of fractional-order multiscaling anomalous diffusion. Phys. Rev. E 74, 026706, 10pp. 